\title{
Metodologia para o cálculo dos módulos de elasticidade longitudinal e transversal em vigas de madeira de dimensões estruturais
}

\author{
Methodology used to determine the shear and longitudinal modulus of elasticity in timber beams
}

\author{
André Luis Christoforo ${ }^{\mathrm{I}^{*}}$ Sérgio Luiz Moni Ribeiro Filho ${ }^{\mathrm{I}}$ Túlio Hallak Panzera ${ }^{\mathrm{I}}$ \\ Francisco Antonio Rocco LahriI
}

RESUMO

Este trabalhou objetiva apresentar uma metodologia analítica para o cálculo dos módulos de elasticidade longitudinal (E) e transversal (G) em vigas de madeira de dimensões estruturais, segundo o emprego das teorias de vigas de Euler Bernoulli e Timoshenko, sendo utilizado o ensaio de flexão estática a três pontos. As madeiras testadas foram o Pinus elliottii e a Corymbia citriodora. Os resultados encontrados relevaram ser o módulo de elasticidade longitudinal 18,70 vezes superior ao módulo transversal do Pinus elliottii e 21,2 superior ao módulo transversal do Corymbia citriodora, sendo estes compativeis quando comparada a relação entre $E$ e $G$ estabelecida pela norma Brasileira ABNT NBR 7190:1997 (Projeto de Estruturas de Madeira), que define ser o módulo de elasticidade longitudinal vinte vezes superior ao transversal.

Palavras-chave: madeira, teoria de vigas, módulo de elasticidade.

\section{ABSTRACT}

This paper proposed a test method to obtain the shear $(G)$ and longitudinal $(E)$ modulus of elasticity in timber beams with structural dimensions, based on the static threepoints bending tests and the Euler Bernoulli and Timoshenko beams theories. The woods tested were the Corymbia citriodora and Pinus elliottii. The results revealed that the longitudinal modulus of elasticity of Pinus elliottii is 18.70 greater than the shear modulus, and 21.16 greater than the shear modulus of Corymbia citriodora, being consistent this results when compared to the proposed by the Brazilian standard ABNT NBR 7190:1997 (Design of Wood Structures), being the longitudinal modulus of elasticity twenty times greater than the shear modulus.

Key words: timber, beams theory, modulus of elasticity.

\section{INTRODUÇÃO}

Dentre as principais propriedades requisitadas para o dimensionamento de uma estrutura, destaca-se o módulo de elasticidade. Em se tratando da madeira, por ser este um material natural de grande complexidade anatômica e variabilidade, apresentando três eixos de simetria nas direções radial, tangencial e longitudinal, a obtenção precisa de todos os seus parâmetros elásticos fica comprometida. Entretanto, os projetos estruturais são realizados com posse das suas propriedades mecânicas equivalentes, advindas de ensaios experimentais padronizados por códigos normativos que visam a quantificar tais variáveis às devidas condições de solicitação mecânica.

Em um projeto de vigas, assim como de outros elementos estruturais, o conhecimento dos módulos de elasticidade longitudinal $(E)$ e transversal $(G)$ é de fundamental importância (CHRISTOFORO, 2011). No Brasil, a caracterização da madeira na flexão é feita de acordo com a norma ABNT NBR 7190:1997 (Projeto de Estruturas de Madeira), que preconiza o ensaio de flexão estática a três pontos, restrita a corpos-de-prova de pequenas dimensões e isentos de defeitos, apresentando uma relação empírica para a obtenção do módulo transversal, conhecido o valor do módulo longitudinal, sendo $\mathrm{G}=\mathrm{E} / 20$.

No Brasil, as pesquisas que envolvem a caracterização de peças de madeira de dimensões

'Departamento de Engenharia Mecânica, Universidade Federal de São João del-Rei (UFSJ), Praça Frei Orlando, 170, centro, 36307-352, São João del-Rei (MG). E-mail: alchristoforo@yahoo.com.br. *Autor para correspondência.

IIDepartamento de Engenharia de Estruturas, Escola de Engenharia de São Carlos (EESC), Universidade de São Paulo (USP), São Carlos, SP, Brasil. 
estruturais seguem as premissas de métodos e cálculos contidos em documentos normativos internacionais, podendo-se citar os trabalhos de PIGOZZO et al. (2000), FIORELLI et al. (2009) e MIOTTO (2009).

Metodologias não destrutivas de ensaios têm sido amplamente utilizadas na obtenção do módulo de elasticidade longitudinal em peças de madeira de dimensões estruturais, ressaltandose o uso do ultrassom e da vibração transversal, enfatizando a simplicidade e eficiência do emprego de tais metodologias, justificadas pela possibilidade de uso da peça depois de ensaiada (ROSS et al., 1998; ERIKSON et al., 2000; OLIVEIRA \& SALES, 2002; MINÁ et al., 2004; WANG et al., 2008; LIANG \& FU, 2007; DONG e HAI, 2011; SALES et al., 2011).

Com relação à determinação do módulo de elasticidade transversal, tanto em vigas de madeira como em corpos-de-prova de pequenas dimensões, são poucas as pesquisas desenvolvidas, dentre elas, destacando-se os trabalhos de ROCCO LAHR (1983), BURDZIK \& NKWERA (2002) e ZANGIÁCOMO \& ROCCO LAHR (2008).

ROCCO LAHR (1983) avaliou, além de outras, a influência das dimensões dos corpos de prova de madeira serrada para os quais o efeito das forças cisalhantes se tornam desprezíveis no cálculo dos deslocamentos, chegando à relação $L / h \geq 21$, sendo $L$ o comprimento e $h$ a altura da seção transversal.

BURDZIK \& NKWERA (2002) avaliaram os módulos de elasticidade longitudinal e transversal em vigas de madeira da espécie Eucalyptus grandis pelo método de vibração transversal de ondas. Os resultados demonstraram que o método proposto é empregável na determinação dos módulos de elasticidade, apresentando coerência nos resultados, quando comparados com as propriedades da madeira advindas de documento normativo.

ZANGIÁCOMO \& ROCCO LAHR (2008) estudaram a relação entre o comprimento e diâmetro $(D)$ em vigas roliças de madeira para o qual o efeito das forças cisalhantes se torna desprezível no cálculo dos deslocamentos, chegando às relações 12 e 15 para as madeiras Pinus elliottii e Pinus caribaea, respectivamente.

Em linhas gerais, os ensaios em peças de dimensões estruturais consistem no emprego de métodos não destrutivos como o da vibração transversal e ultrassom, sendo investigados essencialmente o módulo de elasticidade longitudinal. $\mathrm{O}$ presente trabalho objetiva apresentar uma metodologia de cálculo para a obtenção dos módulos de elasticidade transversal e longitudinal em vigas de madeira de dimensões estruturais, fundamentado no ensaio de flexão estática a três pontos e nas teorias de viga de Euler Bernoulli e Timoshenko.

\section{METODOLOGIA}

A metodologia experimental utilizada para o cálculo do $E$ e do $G$ em peças de dimensões estruturais de madeira serrada baseia-se no trabalho de ROCCO LAHR (1983). De forma alternativa, os módulos de elasticidade são obtidos na condição de linearidade geométrica, estando o maior deslocamento no experimento limitado à medida $\mathrm{L} / 300$ ( $\mathrm{L} \mathrm{em} \mathrm{cm}$ ), sendo L/200 a medida de pequenos deslocamentos definida pela norma Brasileira ABNT NBR 7190:1997.

O Método da Força Virtual (MFV) é empregado sobre o modelo estrutural de flexão estática a três pontos, objetivando-se encontrar a expressão para o cálculo do deslocamento abaixo do ponto de aplicação da força (Equação 1), considerando-se, para tanto, a parcela dos esforços momento fletor e cortante, sendo $F$ a força pontual aplicada, $E$ o módulo de elasticidade longitudinal ou módulo de Young, $I$ o momento de inércia da seção transversal, $G$ o módulo de elasticidade transversal, $A$ a área da seção transversal, $L$ o comprimento útil da viga (distância entre apoios) e $b$ e $h$ as medidas da base e da altura da seção transversal, respectivamente.

$\Delta_{B}=\frac{F \cdot L^{3}}{48 \cdot E \cdot I}+\frac{3 \cdot F \cdot L}{10 \cdot G \cdot A}$

Segundo a presente metodologia, para o cálculo dos módulos de elasticidade, é necessária a execução de dois ensaios experimentais sucessivos sobre a mesma peça. No primeiro, admitindo-se o comprimento da peça $L_{l}$, com $L_{l} / h \geq 21$ (ROCCO LAHR, 1983), determina-se o valor da força $\mathrm{F}_{1}$, responsável por provocar um deslocamento $\delta_{1}=L_{1} / 300$. No segundo ensaio de flexão, os apoios são aproximados, conferindo um novo comprimento útil para a peça $\left(L_{2}\right)$, devendo $L_{2}$ respeitar a desigualdade $L_{2} / h \geq 5 / 4$ (SANCHES Jr \& VENTURINI, 2006), garantindo que as seções permaneçam planas depois de deformadas, obtendo-se um valor de força $\left(F_{2}\right)$ responsável por provocar um deslocamento de magnitude $\delta_{2}=L_{2} / 300$.

$\mathrm{O}$ emprego das forças $F_{1}$ e $F_{2}$, das medidas $L_{1}$ e $L_{2}$ e dos deslocamentos $\delta_{1}$ e $\delta_{2}$ na Equação 1 conduz a um sistema de equações (Equação 2).

$\left\{\begin{array}{l}\frac{F_{1} \cdot L_{1}^{3}}{48 \cdot I_{z}} \cdot \frac{1}{E}+\frac{3 \cdot F_{1} \cdot L_{1}}{10 \cdot A} \cdot \frac{1}{G}=\delta_{1} \\ \frac{F_{2} \cdot L_{2}^{3}}{48 \cdot I_{z}} \cdot \frac{1}{E}+\frac{3 \cdot F_{2} \cdot L_{2}}{10 \cdot A} \cdot \frac{1}{G}=\delta_{2}\end{array}\right.$ 
Sabendo-se que a área e o momento de inércia da seção transversal retangular são respectivamente iguais $b \cdot h$ e $b \cdot h^{3} / 12$ e resolvendo-se o sistema da Equação 2 chega-se ao valor dos módulos de elasticidade $E$ e $G$, expressos, respectivamente, pelas Equações 3 e 4 .

$$
\begin{aligned}
& E=\frac{F_{1} \cdot F_{2} \cdot L_{1} \cdot L_{2} \cdot\left(L_{1}^{2}-L_{2}^{2}\right)}{4 \cdot b \cdot h^{3} \cdot\left(F_{2} \cdot L_{2} \cdot \delta_{1}-F_{1} \cdot L_{1} \cdot \delta_{2}\right)} \\
& G=\frac{3 \cdot F_{1} \cdot F_{2} \cdot L_{1} \cdot L_{2} \cdot\left(L_{1}^{2}-L_{2}^{2}\right)}{10 \cdot b \cdot h \cdot\left(F_{1} \cdot L_{1}^{3} \cdot \delta_{2}-F_{2} \cdot L_{2}^{3} \cdot \delta_{1}\right)}
\end{aligned}
$$

Como a determinação dos módulos de elasticidade conta com duas distâncias distintas entre os apoios $\left(L_{1}\right.$ e $L_{2}$, com $\left.L_{2}<L_{1}\right)$, o segundo ensaio conduz a dois balanços nas extremidades livres da peça. Com o intuito de se comprovar a pequena influência do peso próprio no cálculo dos deslocamentos (ponto médio da viga) pela variação dos apoios, foram desenvolvidas, segundo o emprego do MFV, expressões analíticas para o cálculo dos deslocamentos no ponto médio $\left(\delta_{m}\right)$ e no balanço $\left(\delta_{b}\right)$, expressas, respectivamente, pelas Equações 5 e 6 , sendo $L$ o comprimento útil da peça, $q$ a força uniformemente distribuída (peso próprio) e $c$ o balanço (Figura 1).

$$
\delta_{m}=\frac{L^{2} \cdot\left(5 \cdot q \cdot L^{2}+8 \cdot F \cdot L-24 \cdot q \cdot c^{2}\right)}{32 \cdot E \cdot b \cdot h^{3}}
$$

$$
\delta_{b}=\frac{c \cdot\left[q \cdot\left(12 \cdot L \cdot c^{2}-2 \cdot L^{3}+6 \cdot c^{3}\right)-3 \cdot F \cdot L^{2}\right]}{4 \cdot E \cdot b \cdot h^{3}}
$$

Para avaliar a influência do peso próprio, foram considerados os seguintes valores para as variáveis estruturais: $E=16780 \mathrm{MPa} ; \rho=575 \mathrm{~kg} / \mathrm{m}^{3}$; $b=0,06 m, h=0,10 m$ e $F=1 \mathrm{kN}$, sendo investigadas numericamente cinco condições distintas entre comprimento útil e balanço, considerando-se nulo $(S P P)$ e não nulo $(C P P)$ o peso próprio das peças. A tabela 1 apresenta as condições investigadas $(C d)$, os valores dos deslocamentos no ponto médio e no balanço e o erro percentual relativo $(e r)$ entre os deslocamentos no ponto médio, considerando $(C P P)$ e desconsiderando (SPP) a ação do peso próprio.

Os resultados apresentados na tabela 1 entre os deslocamentos no ponto médio, considerando-se ou não a existência do peso próprio, comprovam sua pequena influência, sendo o maior erro encontrado de $1,34 \%$, referente à condição 1 , validando-se, dessa forma, o emprego das Equações 3 e 4, propostas para o cálculo dos módulos de elasticidade $E$ e $G$.

A metodologia proposta e discutida foi empregada em 14 peças estruturais de madeira de dimensões $4 \mathrm{~cm} \times 5 \mathrm{~cm} \times 140 \mathrm{~cm}$, ensaiadas na posição de menor inércia (flatwise) e posicionadas no plano LR (Longitudinal e Radial), sendo sete peças da espécie Corymbia citriodora e outras sete da espécie Pinus elliottii, obtidas da empresa Serraria Agostini da cidade de São João del-Rei (MG), plantadas e extraídas (com idade média de sete anos) na região da cidade de Salinas (MG). No primeiro ensaio, o comprimento útil $\left(L_{1}\right)$ das peças é de $136 \mathrm{~cm}$ (distância entre apoios), sendo de $80 \mathrm{~cm}$ a distância entre apoios $\left(L_{2}\right)$ utilizada no segundo ensaio.

Dos testes preliminares executados, contatou-se que o comprimento $L_{2}$ deve ser no mínimo $30 \%$ inferior ao comprimento $L_{l}$, conduzindo a um valor estável para o módulo de elasticidade transversal.

Objetivando relacionar os valores dos módulos de elasticidade $E$ e $G$ para as espécies avaliadas e posterior comparação com a relação

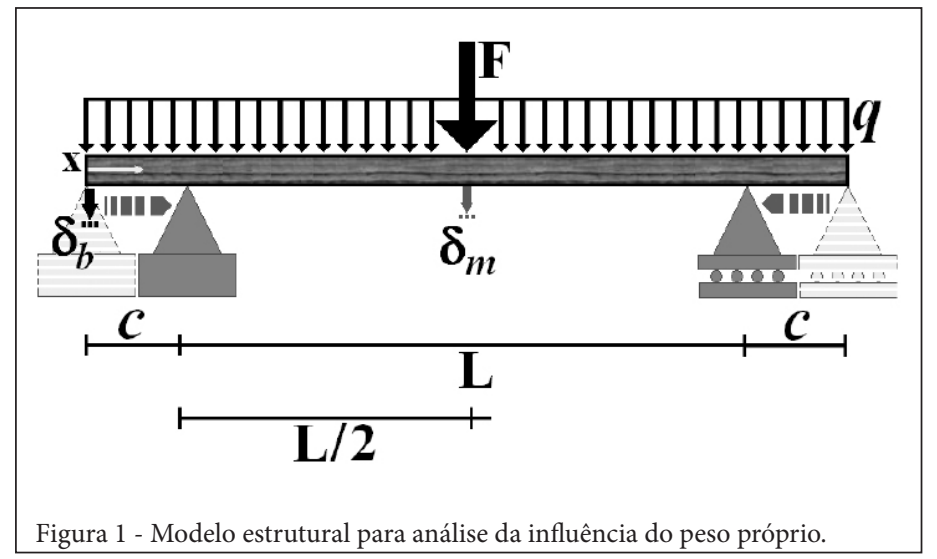

Ciência Rural, v.43, n.4, mar, 2013. 
Tabela 1 - Deslocamentos no ponto médio e no balanço $(\mathrm{mm})$.

\begin{tabular}{|c|c|c|c|c|c|c|c|c|}
\hline \multirow{2}{*}{$C d$} & \multirow{2}{*}{$L(m)$} & \multirow{2}{*}{$c(\mathrm{~m})$} & \multirow{2}{*}{$L / h$} & \multicolumn{2}{|c|}{-------------- $C P P$------------- } & \multicolumn{2}{|c|}{--------------SPP------------- } & \multirow{2}{*}{ er $(\%)$} \\
\hline & & & & $\delta_{m}(\mathrm{~mm})$ & $\delta_{b}(\mathrm{~mm})$ & $\delta_{m}(\mathrm{~mm})$ & $\delta_{b}(\mathrm{~mm})$ & \\
\hline 1 & 4,0 & 0,0 & 40 & $16,11 \downarrow$ & $\mathrm{X}$ & $15,89 \downarrow$ & $\mathrm{X}$ & 1,34 \\
\hline 2 & 3,5 & 0,25 & 35 & 10,77 & $2,31 \uparrow$ & $10,65 \downarrow$ & $2,28 \uparrow$ & 1,13 \\
\hline 3 & 3,0 & 0,50 & 30 & 6,77 & $3,38 \uparrow$ & $6,70 \downarrow$ & $3,35 \uparrow$ & 1,05 \\
\hline 4 & 2,5 & 0,75 & 25 & 3,90 & $3,50 \uparrow$ & 3,88 & $3,49 \uparrow$ & 0,52 \\
\hline 5 & 2,0 & 1,00 & 20 & 1,98 & $2,98 \uparrow$ & 1,99 & $2,98 \uparrow$ & 0,50 \\
\hline
\end{tabular}

definida pela norma Brasileira ABNT NBR 7190:1997, foi utilizado o método dos mínimos quadrados, expresso pela Equação 7, sendo $\alpha$ o coeficiente a ser ajustado pelo critério do menor resíduo $(E=\alpha \cdot G)$.

$f(\alpha)=\frac{1}{2} \cdot \sum_{i=1}^{n}\left(E_{i}-\alpha \cdot G_{i}\right)^{2}$

\section{RESULTADOS}

A tabela 2 apresenta os resultados obtidos para os módulos de elasticidade longitudinal e transversal das peças de madeira das espécies Corymbia citriodora e Pinus elliottii.

A figura 2 ilustra os resultados do teste de normalidade de Anderson-Darling sobre os valores dos módulos de elasticidade longitudinal e transversal para as espécies de madeira aqui avaliadas, sendo os P-valores encontrados ambos superiores a 0,05 (5\%), comprovando a normalidade dos dados avaliados (MONTGOMERY, 2009).

Os valores encontrados do coeficiente $\alpha$ para as madeiras Pinus elliottii e Corymbia citriodora pelo critério de mínimos quadrados são, respectivamente, 18,70 e 21,16 , sendo $6,50 \%$ inferior e $5,80 \%$ superior ao coeficiente da relação entre $E$ e $G$, proposto pelo documento normativo ABNT NBR 7190:1997.

\section{CONCLUSÃO}

A presente metodologia mostrou ser de simples empregabilidade, conduzindo a relações entre os módulos de elasticidade longitudinal e transversal das madeiras avaliadas próximas à estabelecida pela norma Brasileira ABNT NBR 7190:1997. Ressaltase que os resultados apresentados não podem ser generalizados em função da quantidade de corpos de prova utilizados, sendo necessários novos estudos.

A restrição dos deslocamentos no ensaio de flexão (L/300) confere à presente metodologia caráter não destrutivo, mostrando-se interessante pela possibilidade de uso da peça depois de ensaiada.

A restrição do maior comprimento $L_{1}$ ser no mínimo igual a vinte e uma vezes o valor da altura da seção transversal da peça garante a validade do emprego da teoria de vigas de Euler Bernoulli, possibilitando desprezar o efeito das forças cisalhantes no cálculo dos deslocamentos referente ao primeiro ensaio $\left(L_{l}\right)$. Esse resultado por sua vez comprova ser válido o uso do ensaio estrutural de flexão estática a três pontos, mesmo sabendo ser o esforço cortante não nulo na região de aplicação da carga.

O estudo analítico da influência do peso próprio no cálculo dos deslocamentos no ponto médio das vigas para a condição de balanço (segundo ensaio) foi capaz de comprovar a sua pequena relevância, validando-se, dessa forma, a metodologia de cálculo aqui apresentada.

Tabela 2 - Valores dos módulos de elasticidade longitudinal e transversal.

\begin{tabular}{|c|c|c|c|c|c|}
\hline Pinus elliottii & $\mathrm{E}(\mathrm{MPa})$ & $\mathrm{G}(\mathrm{MPa})$ & Corymbia citriodora & $\mathrm{E}(\mathrm{MPa})$ & $\mathrm{G}(\mathrm{MPa})$ \\
\hline 1 & 12170 & 710 & 1 & 15870 & 860 \\
\hline 2 & 13193 & 772 & 2 & 17474 & 903 \\
\hline 3 & 10246 & 543 & 3 & 21715 & 1084 \\
\hline 4 & 11350 & 622 & 4 & 20280 & 873 \\
\hline 5 & 13467 & 657 & 5 & 19728 & 845 \\
\hline 6 & 12387 & 596 & 6 & 16932 & 741 \\
\hline 7 & 11208 & 572 & 7 & 21457 & 979 \\
\hline
\end{tabular}

Ciência Rural, v.43, n.4, mar, 2013. 


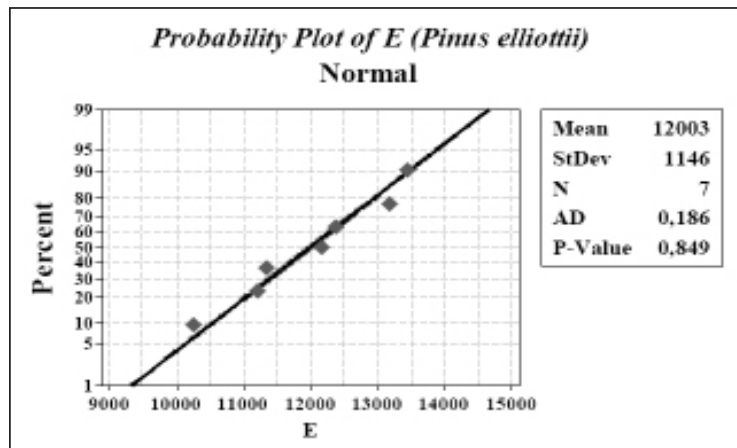

(a)

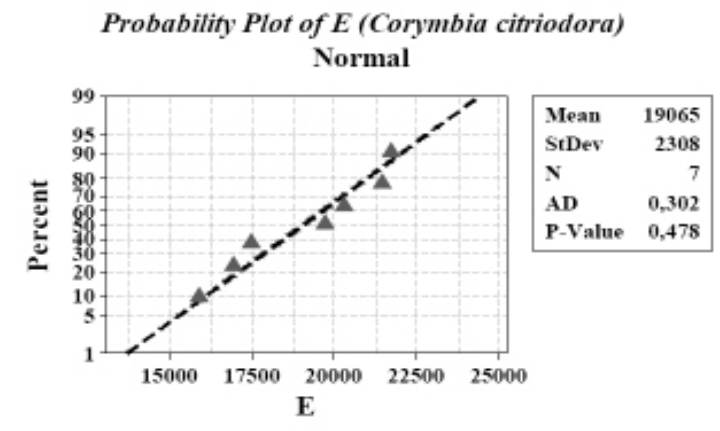

(c)

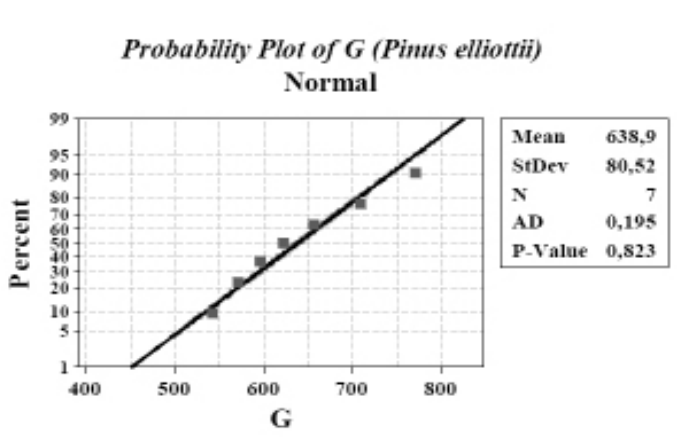

(b)

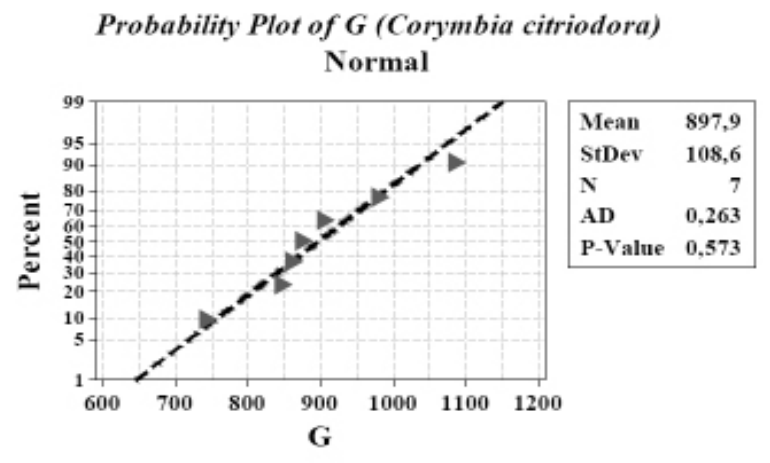

(d)

Figura 2 - Teste de normalidade para os valores dos módulos de elasticidade.

O teste de Anderson-Darling comprovou ser normal a distribuição dos valores encontrados para os módulos de elasticidade de ambas as espécies investigadas, validando os resultados dos coeficientes $\alpha$ encontrados.

Ressalta-se que as peças de madeira neste trabalho foram posicionadas segundo o plano Longitudinal-Radial. Pelo comportamento ortotrópico da madeira, os valores dos coeficientes $\alpha$ podem ser diferentes mediante a escolha do posicionamento das fibras, podendo também diferir para madeiras da mesma ou de outras espécies e com outras dimensões, justificando, dessa forma, o emprego da metodologia elaborada em cada estudo desenvolvido.

\section{REFERÊNCIAS}

ASSOCIAÇÃO BRASILEIRA DE NORMAS TÉCNICAS (ABNT) - NBR 7190. Projeto de estruturas de madeira. Rio de Janeiro, 107p.,1997.

BURDZIK, W.M.G.; NKWERA, P.D. Transverse vibration tests for prediction of stiffness and strength properties of full size Eucalyptus grandis. Forest Products Journal, v.52, n.6, p.6367, 2002.
CHRISTOFORO, A.L. et al. Numerical evaluation of the longitudinal modulus of elasticity in structural round timber elements of Eucalyptus genus. Revista Engenharia Agrícola, v.31, p.1007-1014, 2011.

DONG, X.H.; HAI, W.L. Comparative study on four different methods for measuring the dynamic modulus of elasticity of Acer mono wood. Advanced Materials Research, v.160-162, p.384388, 2011.

ERIKSON, R.G. et al. Mechanical grading of lumber sawn from small-diameter lodgepole pine, ponderosa pine, and grand fir trees from northern Idaho. Forest Products Journal, v.50, n.7-8, p.5965,2000 .

FIORELLI, J. et al. Propriedades mecânicas de peças com dimensões estruturais de Pinus spp: correlação entre resistência à tração e classificação visual. Revista Árvore (Impresso), v. 33, n. 4, p.741-750, 2009.

LIANG, S.; FU, F. Comparative study on three dynamic modulus of elasticity and static modulus of elasticity for Lodgepole pine lumber. Journal of Forestry Research, v.18, p.309-312, 2007.

MINÁ, A.J.S. et al. Avaliação não destrutiva de postes de madeira por meio de ultrassom. Scientia Forestalis, n.65, p.188-196, 2004.

MIOTTO, J.L.; DIAS, A.A. Produção e avaliação de vigas de madeira laminada colada confeccionadas com lâminas de

Ciência Rural, v.43, n.4, mar, 2013. 
Eucalipto. Revista Tecnológica, Edição Especial ENTECA, p.3545, 2009.

MONTGOMERY, D.C. Design and analysis of experiments. $7^{\mathrm{a}}$ ed. Hoboken, Arizona: John Wiley \& Sons, 260 p., 2009.

OLIVEIRA, F.G.R.; SALES, A. Ultrassonic measurements in Brazilian hardwood. Materials Research, v.5, n.1, p.51-55, 2002.

PIGOZZO, J.C. et al. Aspectos da classificação mecânica de peças estruturais de madeira. In: In: VII Encontro Brasileiro em Madeiras e em Estruturas de Madeira, Anais CD-Rom, São Carlos- SP., 2000.

ROCCO LAHR, F.A. Sobre a determinação de propriedades de elasticidade da madeira. 1983. 216f. Tese (Doutorado em Engenharia de Estruturas) - Escola de Engenharia de São Carlos, Universidade de São Paulo, São Carlos, SP.
ROSS. R.J. et al. Nondestructive evaluation of wood. Forest Products Journal, v.48, n.1, p.14-19, 1998.

SALES, A. et al. Evaluation of the mechanical properties of Brazilian lumber (Goupia glabra) by nondestructive techniques. Construction and Building Materials, v.25, n.3, p.1450-1454, 2011.

SANCHES Jr., F.; VENTURINI, W.S. Damage modelling of reinforced concrete beams. Advances in Engineering Software, v.38, n.8-9, p.538-546, 2006.

WANG, S.Y. et al. Grading of softwood lumber using nondestructive techniques. Journal of Materials Processing Technology, v.208, p.149-158, 2008

ZANGIÁCOMO. A. L.; ROCCO LAHR, F.A. Avaliação do efeito do cisalhamento na flexão de elementos roliços da espécie Eucalyptus Citriodora. In: XI Encontro Brasileiro em Madeiras e em Estruturas de Madeira, Anais CD-Rom, Londrina-PR., 2008. 